- Part Three provides useful reference information, a glossary of terms and definitions and a directory of useful organisations.

While considering the need for the pathway, it became apparent that an additional resource of an information leaflet for families was required which will help them reach an informed decision about the care they wish their child to receive.

\title{
P2 A CARE PATHWAY TO SUPPORT EXTUBATION WITHIN A CHILDREN'S PALLIATIVE CARE FRAMEWORK
}

Katrina McNamara ACT, Bristol UK

The need for this work was identified by a number of clinicians who asked ACT to develop a resource following an initial trawl for information about existing guidance and resources via the online global forum PaedPalCare.

This guidance on best practice for professionals in supporting families' decision making and the practical aspects of extubation, will lead to an improvement in the standards of care delivery and the choice offered to families. There is also an information leaflet for families which will help them reach an informed decision about the care they wish their child to receive.

The resource is appropriate for use in a variety of settings, including critical care, palliative care and community settings such as the child's home or a children's hospice. There are three sections to the resource

- Part One provides an introduction to children's palliative care and ACT's care pathway approach. It discusses the need for a dedicated pathway approach and outlines the principles that ACT recommends in adopting and delivering the care pathway to support extubation within a children's palliative care framework.

- Part Two takes the reader through the pathway to support extubation within a children's palliative care framework. There are three stages to the pathway: the journey towards a decision to withdraw life-sustaining ventilation; the practicalities of withdrawing life-sustaining ventilation; and care at the time of death. Each stage of the pathway includes sentinel standards: key goals and useful resources to support care. 